Itinéraires Itinéraires

Littérature, textes, cultures

\title{
Bolivarien : un adjectif porteur de mémoire ? Du souvenir à l'oubli du Libertador
}

Églantine Samouth

\section{OpenEdition}

Journals

Édition électronique

URL : http://journals.openedition.org/itineraires/145

DOI : 10.4000/itineraires.145

ISSN : 2427-920X

Éditeur

Pléiade

\section{Édition imprimée}

Date de publication : 1 juillet 2011

Pagination : 97-11

ISBN : 978-2-296-54673-8

ISSN : 2100-1340

Référence électronique

Églantine Samouth, "Bolivarien : un adjectif porteur de mémoire? Du souvenir à l'oubli du Libertador », Itinéraires [En ligne], 2011-2 | 2011, mis en ligne le 03 février 2015, consulté le 19 avril 2019. URL :

http://journals.openedition.org/itineraires/145; DOI : 10.4000/itineraires.145

\section{(c) $\oplus \Theta \Theta$}

Itinéraires est mis à disposition selon les termes de la licence Creative Commons Attribution - Pas d'Utilisation Commerciale - Pas de Modification 4.0 International. 


\title{
Bolivarien : un adjectif porteur de mémoire? Du souvenir à l'oubli du Libertador
}

\begin{abstract}
Does the adjective bolivariano, derived from the proper name Simón Bolívar, carry a memory connected to this great name? This adjective is ambiguous - due to its morphology - and President Hugo Chávez uses it abundantly, which allows him to mobilize the positive values that are associated in the collective memory with this mythical figure. Then, when circulating within the Venezuelan press, the adjective bolivariano retains traces of these usages and takes on a new meaning, becoming associated with the figure of Hugo Chávez from then on.
\end{abstract}

Keywords : Venezuelan political speech, adjective bolivariano, proper name, collective memory, dys-memory

Mots clés : discours politique vénézuélien, adjectif bolivariano, nom propre, mémoire collective, dé-mémoire

L'étude présentée ici a pour point de départ une observation effectuée lors de l'analyse d'un corpus de presse vénézuélienne constitué autour de la tentative de coup d'Etat contre Hugo Chávez, en avril 2002 : on y relève une certaine profusion d'emplois de l'adjectif bolivariano, dans des contextes variés où la relation avec Simón Bolívar semble parfois plus que lointaine. Si la référence à ce héros de l'indépendance latino-américaine a été une constante dans l'histoire politique du pays, avec l'élection d'Hugo Chávez à la présidence de la République en 1998, elle est devenue incontournable. Par conséquent, il nous a semblé que cet adjectif pouvait constituer un lieu d'observation de l'héritage du mythe bolivarien dans le Venezuela d'aujourd'hui, et plus particulièrement, dans le langage politique de ce pays. On peut se demander en effet dans quelle mesure cet adjectif est porteur d'une mémoire liée au personnage illustre auquel renvoie le nom propre dont il dérive. Nous reviendrons donc tout d'abord rapidement sur l'importance de la figure du Libertador au Venezuela et sur l'utilisation de ce terme par Hugo Chávez, avant de nous intéresser à 
1'histoire et à la morphologie de 1'adjectif bolivariano lui-même. Notre troisième partie sera quant à elle dédiée à l'analyse des emplois de bolivariano dans le corpus de presse mentionné précédemment.

\section{Le Venezuela : un pays « bolivarien »}

Resté célèbre pour son engagement dans les combats pour l'indépendance de plusieurs pays d'Amérique latine, dont sa terre natale, le Venezuela, Simón Bolívar est ainsi devenu le symbole de la fondation de cette nation. La vénération populaire dont il fait l'objet dans ce pays ainsi que l'utilisation politique de sa figure ont conduit de nombreux auteurs à parler d'un " culte de Bolívar », un culte que l'historien vénézuélien Germán Carrera Damas (1989 : 290) décrit comme un culte « $d u$ peuple » et «pour le peuple». Ce culte prend une dimension institutionnelle peu de temps après la mort du Libertador (en 1833) avec le rapatriement en 1842 de sa dépouille mortelle de Colombie au Venezuela, et continuera d'être entretenu par les gouvernements successifs au cours des XIX et $\mathrm{XX}^{\mathrm{e}}$ siècles. Bolívar, écrit Inés Quintero (2000 : 15), est « le symbole des vertus républicaines, le modèle impérissable des Vénézuéliens ».

Ainsi, lorsqu'en 1982 le commandant Hugo Chávez fonde avec quelques autres militaires l'Armée Bolivarienne Révolutionnaire (Ejército Bolivariano Revolucionario), groupe clandestin se revendiquant des idéaux du Père de la Patrie, il n'est pas le premier à raviver le « mythe bolivarien ». Mais après son accession au pouvoir, en 1998, la référence au héros devient omniprésente. Une nouvelle constitution est approuvée en 1999, dont les principes se fondent « sur la doctrine de Simón Bolívar, le Libérateur ${ }^{1}$ ». Se présenter comme l'héritier de l'œuvre indépendantiste du Libertador est en effet le mode de légitimation suprême pour l'entreprise de refondation de la nation du nouveau président. Dans son discours, les références à Simón Bolívar abondent, apparaissant sous des formes multiples, telles par exemple, des citations que le président récite de mémoire :

\footnotetext{
Je termine par cette phrase de Bolívar, qui fait partie du serment que nous avons fait il y a des années : « Je ne donnerai de repos ni à mon bras ni à mon âme, nous ne connaîtrons de répit que lorsque nous verrons le Venezuela véritablement tel que nous l'avons rêvé, digne, prospère, glorieux ${ }^{2}$.»
}

1. Voir l'article 1 de la Constitución de la República Bolivariana de Venezuela de 1999.

2. «Me despido con esta frase de Bolívar, que forma parte del juramento que hicimos años atrás : "No daré descanso a mi brazo ni reposo a mi alma, no descansaremos hasta que veamos a Venezuela verdaderamente como la soñamos, digna, próspera, gloriosa." ", Alocución con motivo de los cien primeros días de gobierno, http://www.analitica.com/ bitblioteca/hchavez/100dias.asp. Tous les sites Internet ont été consultés le 21 juin 2010. Nous traduisons toutes les citations. 
De plus, certains auteurs vénézuéliens ${ }^{3}$ soulignent qu'Hugo Chávez a réintroduit dans le lexique politique certains termes datant de la période de 1'Indépendance, tels mantuano, qui désignait à cette époque les membres de l'élite créole, ou oligarquía que le président vénézuélien utilise fréquemment en référence à ses adversaires politiques :

Vous savez que de son vivant Bolívar fut trahi par l'oligarchie déprédatrice, cette même oligarchie qui veut maintenant se pavaner, cette même oligarchie qui maintenant menace de manière ridicule le gouvernement révolutionnaire ${ }^{4}$.

La période indépendantiste et le vocabulaire qui lui est associé semblent donc former la « deixis fondatrice » du discours d'Hugo Chávez, c'est-à-dire « la ou les situations d'énonciation antérieures dont la deixis actuelle se donne pour la répétition et dont elle tire une bonne part de sa légitimité » (Maingueneau $1991: 113$ ).

D'autre part, et c'est ce qui nous intéresse ici, le président vénézuélien qualifie de « bolivarien » tout ce qui a trait à son projet politique, et une bonne partie des initiatives menées par son gouvernement. Dans la constitution approuvée à son arrivée au pouvoir, et dont il est le principal artisan, la République du Venezuela est ainsi rebaptisée « République bolivarienne du Venezuela ». Le texte lui-même, dont le nom officiel est Constitution de la République bolivarienne du Venezuela, devient, dans ses discours, la "Constitution bolivarienne ». Dés le début de son mandat, il appelle les citoyens vénézuéliens à constituer des « cercles bolivariens», organisations locales dont la mission est de mener une action sociale en défendant les idéaux de Simón Bolívar et du nouveau gouvernement ${ }^{5}$. Il appelle à créer des « écoles bolivariennes », puis, en 2003, fonde l'Université bolivarienne $\mathrm{du}$ Venezuela. Tout cela prend place dans le projet politique qu'il entend mener à bien, celui de la « révolution bolivarienne ».

Il fait dans ses discours un usage immodéré de ce terme. On y trouve ainsi de nombreux emplois substantivés : "Voici notre frère Percibal Patterson, Premier ministre de Jamaïque, c'est un bolivarien, un bolivarien, Patterson $^{6} »$. Dans cet exemple, bolivariano semble indiquer l'adhésion à une doctrine politique : celle du bolivarianisme. À plusieurs reprises en

3. Voir Irma Chumaceiro Arreaza (2003 : 33) et Luis Barrera Linares (2003: 64-65).

4. «Ustedes saben que Bolívar fue traicionado en vida por la oligarquía depredadora, esta misma oligarquía que ahora quiere cantar como gallos, esta misma oligarquía que ahora amenaza de manera ridícula al gobierno revolucionario », Discurso en la juramentación de los Círculos Bolivarianos en Caracas, 17 décembre 2001. Extrait de discours cité dans Irma Chumaceiro Arreaza (2003: 34).

5. Voir http://portal.gobiernoenlinea.ve/cartelera/CirculosBolivarianos.html.

6. «Ahí está nuestro hermano Percibal Patterson, primer ministro de Jamaica, es un bolivariano, un bolivariano, Patterson », Cadena nacional de radio y televisión, 15 juin 2001, http://www.analitica.com/bitblioteca/hchavez/cadena20010615.asp. 
effet, dans le discours d'Hugo Chávez, le terme bolivarien semble devenir un équivalent de « révolutionnaire ": «Non, nous les bolivariens, nous les révolutionnaires nous sommes des humanistes ${ }^{7} \gg$. On relève également des emplois plus inattendus, comme celui-ci, où Hugo Chávez va jusqu'à qualifier Simón Bolívar lui-même de bolivarien : " l'habitant immortel de Caracas, le bolivarien, le révolutionnaire, le leader de tous les temps, le commandant éternel de cette révolution, qui n'est autre que Simón Bolívar $^{8}$ ». Enfin, le président n'hésite pas à saluer ses citoyens d' « une embrassade bolivarienne ${ }^{9} \gg$. Après ces quelques exemples des diverses utilisations qu'en fait Hugo Chávez, on comprendra que le terme bolivariano mérite que l'on se penche sur son signifié.

\section{Un adjectif ambigu}

Nous avons donc mené des recherches dans divers ouvrages et dictionnaires ${ }^{10}$ et sur Internet, afin d'observer quel usage était fait de bolivariano avant qu'Hugo Chávez n'en généralise 1'emploi à des contextes aussi variés, et de tenter de déterminer quelle était son acception « d'origine ». Toutefois, nous n'avons pu établir avec exactitude la date d'apparition du terme. La tenue, en 1926, d'un Congreso bolivariano ${ }^{11}$ (Congrès bolivarien) au Panamá, en commémoration du centenaire du Congrès de Panamá - au cours duquel Simón Bolívar avait appelé les pays alors indépendants à l'unité continentale - est la plus ancienne occurrence du terme que nous ayons relevée. La rubrique « Nouveau trésor lexicographique de la langue espagnole » du dictionnaire en ligne de la Real Academia Española, indique que ce terme a été accepté dans ce dictionnaire en 1936. Celui-ci en donnait alors la définition suivante (qui est restée la même) :

7. « No, nosotros los bolivarianos, nosotros los revolucionarios somos humanistas », «Alocución a la nación al retornar al poder », 14 avril 2002, http://www.analitica.com/ bitblioteca/hchavez/vuelta_al_poder.asp.

8. «[...] el caraqueño inmortal, el bolivariano, el revolucionario, el líder de todos los tiempos, el comandante eterno de esta revolución que no es otro que Simón Bolívar », Juramentación del Comando de la Revolución. Primer año del Gobierno Revolucionario, 10 janvier 2002. Extrait de discours cité par Irma Chumaceiro Arreaza (2003 : 29).

9. «Un abrazo bolivariano ». Voir le discours d'investiture d'Hugo Chávez, le 2 février 1999, http://www.analitica.com/Bitblio/hchavez/toma.asp.

10. Nous avons consulté pour cela des dictionnaires de langue espagnole, des dictionnaires étymologiques, historiques et d'hispanismes, dont les principales références figurent en bibliographie.

11. Voir Ernesto Castillero (1995 : 225). Voir également les liens suivants : http://mensual. prensa.com/mensual/contenido/2006/07/23/hoy/vivir/677782.html ; http://bdigital.binal. ac.pa/bdp/descarga.php?f=accion-3.pdf ; http://ecotropicos.saber.ula.ve/db/ssaber/Edocs/ pubelectronicas/mundouniversitario/num14_enero2005/articulo2.pdf. 


\begin{abstract}
Qui appartient ou est relatif à Simón Bolívar, militaire vénézuélien, ou à son histoire, sa politique, etc. Congrès bolivarien. Doctrine bolivarienne ${ }^{12}$.
\end{abstract}

Peu après, en 1938, le président vénézuélien Eleazar López Contreras, créait la Sociedad bolivariana de Venezuela (la Société bolivarienne du Venezuela), chargée de diffuser les idéaux du Libertador, au travers, par exemple, d'une réédition de l'ensemble de ses écrits (Carrera Damas 1989 : 244).

Parmi les divers emplois que nous avons pu rencontrer après cette date, nous n'en citerons que quelques-uns. L'historien Carrera Damas, dans son ouvrage cité précédemment sur le « culte de Bolívar » l'utilise à maintes reprises, mentionnant par exemple « la politique bolivarienne du général Eleazar López Contreras » (1989 : 245). On y trouve aussi en bibliographie, la référence d'un livre intitulé Culto bolivariano (Culte bolivarien), paru en $1940^{13}$. On relève enfin quelques occurrences du terme bolivariano dans le discours politique, comme dans ce discours du futur président Rómulo Betancourt, lors de la création du parti Action Démocratique en 1941 :

Et travaillons, avec une authentique ferveur bolivarienne - pas cette ferveur bolivarienne d'apparat que nous connaissons tous et que nous répudions $[\ldots]$ - à la réalisation d'une fédération d'États latino-américains ${ }^{14}$.

Ainsi, l'adjectif bolivariano n'est pas une création lexicale ni une spécificité de langage d'Hugo Chávez. Toutefois, ces différents usages du terme ne nous éclairent pas véritablement quant à son acception précise. Pour « Société bolivarienne du Venezuela » et " Culte bolivarien », il semble pouvoir être paraphrasé, simplement, par de Bolívar. Mais dans " Congrès bolivarien », doit-il être interprété comme congrès en hommage à Simón Bolívar, comme congrès prônant les idéaux de Bolívar, comme congrès réunissant des partisans de Bolívar? Par « ferveur bolivarienne », doit-on entendre ferveur digne de celle de Bolivar et de son projet d'union continentale ou ferveur des véritables héritiers de Bolivar?

Un détour par sa morphologie nous semble donc s'imposer ici. L'adjectif bolivariano, tout d'abord, appartient à la classe des adjectifs dérivés de noms, les adjectifs dénominaux. Or, comme le souligne Alice KriegPlanque, un problème se pose pour les adjectifs de ce type, car « ils ne font pas apparaître de façon univoque la relation qu'ils établissent entre le nom dont ils dérivent et le nom qui les régit dans l'énoncé » (2002: 103). L'adjectif bolivariano pose ainsi la question du type de relation qu'il établit

12. «bolivariano, na. adj. Perteneciente o relativo a Simón Bolívar, militar venezolano, o a su historia, su política, etc. Congreso bolivariano. Doctrina bolivariana».

13. Antonio Arraíz 1940. Voir références en bibliographie.

14. «Y trabajemos, con auténtico fervor bolivariano - no ese fervor bolivariano de prestado que todos conocemos y repudiamos [...] - porque llegue a realizarse una federación de Estados latinoamericanos ». 
entre le nom propre Simón Bolivar et le nom qu'il qualifie. Comme on vient de le voir, celui-ci semble en effet osciller entre une valeur « relationnelle », indiquant une simple relation avec le personnage de Simón Bolívar (la geste bolivarienne = la geste de Bolivar) et une valeur $"$ qualitative ${ }^{15} »$, ouvrant alors la voie à diverses interprétations (telles que la révolution bolivarienne = la révolution présentant des qualités dignes des idéaux de Bolivar, un président bolivarien = un président partisan des idées de Bolivar, etc.).

Ceci nous conduit à nous demander si le suffixe -iano qui, adjoint au nom propre de Bolivar, forme l'adjectif bolivariano, ne pourrait pas nous éclairer quant à la relation établie entre ce nom propre et le nom recteur. À propos de ce suffixe, la grammaire descriptive de la langue espagnole de Bosque et Demonte (1999: $\S 70.3 .1)$ indique qu'il constitue le suffixe « par défaut ${ }^{16} \gg$ des adjectifs relationnels dérivés de noms propres de personne. C'est dans ce domaine qu'il est le plus productif, permettant ainsi de former de nombreux d'adjectifs à partir de noms propres : freudiano, clintoniano, ciceroniano, sanmartiniano, etc. Cet ouvrage indique néanmoins que dans le domaine politique, le suffixe -iano entre en concurrence avec le suffixe -ista dans son statut de suffixe « par défaut» (ibid.). Beaucoup d'adjectifs sont en effet formés de noms de personnalités politiques et du suffixe ista: marxista, franquista, castrista, ou chavista. On trouve également cette concurrence en français, au sein des dérivés d'anthroponymes, entre les suffixes équivalents -ien et -iste. Le premier permet de construire des adjectifs « relatifs à la personne dont le nom sert de base à l'adjectif : gaullien "relatif à la personne de de Gaulle" », et le second, des adjectifs " à valeur de partisan de la personne dont le nom sert de base à l'adjectif ou du courant politique dont elle est le représentant emblématique : gaulliste "relatif à de Gaulle en tant que représentant d'une certaine politique" » (Lignon 2002).

Pour Stéphanie Lignon, le suffixe -ien est un suffixe « neutre, non marqué », contrairement à d'autres, tels que -iste, " dont la valeur sémantique est très marquée ». Cette auteure souligne ainsi la propension des adjectifs en -ien à tendre vers des emplois « qualifiants »-par exemple, des emplois de type partisan (stalinien, chiraquien) -, propension qui serait due à l'aspect " malléable » de ce suffixe. Dans les emplois "à valeur de partisan ", cette valeur serait donc imputable, selon elle, " non pas au suffixe lui-même (comme c'est le cas avec le suffixe -iste) mais à la capacité référentielle du nom de base » (ibid.). De ce fait, d'un point de vue morphologique, bolivariano n'a pas de valeur spécifiquement politique ou partisane, valeur qu'il aurait eu d'emblée s'il avait été formé avec le suffixe -ista. Néanmoins, le suffixe-iano ne lui interdit pas d'avoir ce type

15. Pour la distinction entre adjectifs relationnels et adjectifs qualificatifs, voir par exemple la grammaire de Martin Riegel (1994 : 355-356).

16. Par " suffixe par défaut », les auteurs entendent " un suffixe qui peut s'appliquer chaque fois qu'il n'y a pas de suffixe synonyme plus spécifique, plus approprié » (Bosque et Demonte 1999 : 4611, note 21). 
d'acception. Autrement dit, le suffixe-iano ne nous donne pas d'indication particulière quant à la relation qu'établit l'adjectif bolivariano entre le nom qui lui est sous-jacent, Bolívar, et le nom recteur.

D'autre part, l'adjectif bolivariano dérive d'un nom propre, catégorie que les grammaires considèrent bien souvent comme n'ayant pas de signifié en soi. Celle de Riegel indique par exemple que les noms propres sont « dépourvus de sens lexical [...] et ne sont pas susceptibles d'une définition au sens ordinaire du terme » (1994:175-176). Face à cette conception asémantique du nom propre, il existe cependant une autre approche, introduite en linguistique par Paul Siblot (1987), postulant une « signifiance » du nom propre ${ }^{17}$. Selon cette approche, le nom propre produit du sens, qui ne peut être observé qu'en discours. Dans leur introduction au numéro des Carnets du Cediscor sur « le nom propre en discours », Michelle Lecolle, Marie-Anne Paveau et Sandrine Reboul-Touré (2009) parlent ainsi d'une « historicité » du nom propre :

Qu'il s'agisse de nom de lieux, d'événements ou de personnes, que les $\mathrm{Np}$ [noms propres] renvoient à des référents dramatiques ou ludiques, ceux-ci éveillent des échos et construisent des résonances signifiantes, par leur sonorité, par leur monde d'appartenance domanial et par leur pertinence pour des communautés discursives. (19)

Au vu de l'ampleur du mythe « bolivarien » dans la société vénézuélienne, on ne peut que considérer, dans le cas du nom propre Bolivar, que celui-ci fait sens dans la communauté discursive vénézuélienne - et au-delà.

Concernant l'adjectif bolivariano, la question qui se pose alors, comme le font remarquer Paul Siblot et Sarah Leroy (2000) à propos de 1'antonomase - par exemple : « Pierre est un véritable Don Juan »-est celle des représentations auxquelles le nom propre est associé. En effet, dans les cas où bolivariano pourrait recevoir des interprétations qualitatives - comme partisan des idées de Bolivvar ou qui présente des qualités dignes de Simón Bolivar - on peut se demander quels sont les aspects de son histoire, de sa pensée, de son caractère, qui sont retenus comme « représentatifs » de ce personnage. Autrement dit, à quelles valeurs la figure de Simón Bolívar est-elle associée dans la mémoire collective vénézuélienne? Et parmi celles-ci, quelles sont celles véhiculées par l'adjectif bolivariano?

L'adjectif bolivariano est donc ambigu à plusieurs niveaux : d'une part, en tant qu'adjectif dénominal, il est porteur d'une ambiguïté relationnelle; d'autre part, son suffixe neutre ne donne pas d'indication quant à la relation qu'il établit entre le nom de base et le nom qualifié; enfin, en tant que dérivé de nom propre, il pose la question des valeurs associées à la figure de Simón Bolívar. Nous considérons à cet égard, à l'instar d'Alice

17. Sur la mise en cause de la conception asémantique du nom propre, voir également Marc Wilmet (1995) et Kerstin Jonasson (1994). 
Krieg-Planque, que ces ambiguïtés font partie des ressources sémantiques « offertes par la langue à ses utilisateurs » (2002 : 119) et qu'il n'y a pas lieu de les lever. Ainsi, il nous semble que c'est précisément cette non-univocité qui permet à Hugo Chávez d'utiliser si abondamment l'adjectif bolivariano. En effet, lorsqu'il qualifie quelque chose de bolivarien, il ne s'agit pas de le caractériser de manière précise en explicitant quel type de relation est établie avec Bolívar ou quels sont les traits de ce personnage retenus dans cette qualification. Dans les usages qu'en fait le président vénézuélien, l'adjectif bolivariano permet surtout d'évoquer et de mobiliser les valeurs associées à la figure et à l'histoire du Libertador dans la mémoire collective vénézuélienne, valeurs considérées dans ce pays (et même au-delà), comme les plus positives qui soient.

\section{Bolivariano dans la presse vénézuélienne}

Il semble donc intéressant d'étudier la manière dont ce terme, remis au goût du jour par Hugo Chávez, est repris par la presse vénézuélienne. On peut se demander, en effet, si cette dernière l'emploie de manière aussi abondante et si l'adjectif bolivariano est porteur des mêmes valeurs, dans le discours des journaux, que celles qu'il semble véhiculer dans le langage du président. D'autre part, la question qui se pose est celle de l'attitude adoptée par la presse face à l'utilisation de ce terme par Hugo Chávez : est-elle acceptée ou suscite-t-elle le débat? Il s'agit ici d'étudier l'emploi de l'adjectif bolivariano dans le contexte de crise politique majeure que représente la tentative de coup d'État menée contre l'actuel président du Venezuela. En effet, en avril 2002, Hugo Chávez est destitué et remplacé par un président provisoire, Pedro Carmona, dirigeant de l'organisation patronale Fedecámaras, à la suite d'une grève générale et d'importantes manifestations, durant lesquelles plusieurs personnes trouvent la mort. Hugo Chávez recouvre ses fonctions un peu moins de quarante-huit heures plus tard, soutenu par une partie de l'armée, et par les manifestations spontanées de ses partisans. Notre corpus comprend les éditions des trois principaux quotidiens nationaux : El Nacional, El Universal et Últimas Noticias, entre le 12 et le 15 avril 2002, c'est-à-dire à partir de l'annonce dans les journaux de la chute d'Hugo Chávez, jusqu'à celle de son retour au pouvoir. On y relève un total de cent soixante-neuf occurrences de bolivariano, parmi lesquelles se distinguent principalement deux sortes d'emplois, que nous allons analyser ici : d'une part, la reprise de syntagmes créés par Hugo Chávez, et d'autre part ce que nous avons appelé des emplois « libres ».

\section{Un adjectif polémique}

Comme on l'a indiqué précédemment, Hugo Chávez a conçu une série d'expressions intégrant l'adjectif bolivariano. Les plus reprises dans le corpus sont República bolivariana de Venezuela, revolución bolivariana, 
Constitución bolivariana et círculos bolivarianos. Le syntagme República bolivariana de Venezuela, qu'il apparaisse dans du discours direct ou dans le discours du journal, ne fait jamais l'objet de procédés de mise à distance, ce qui peut s'expliquer par le fait qu'il s'agit du nom officiel du pays. Toutefois, l'une des premières mesures du gouvernement de transition mis en place après la chute d'Hugo Chávez est de supprimer l'adjectif bolivariano du nom du pays :

1. La deuxième disposition prise par le président provisoire a été de retirer l'adjectif bolivarien établi par la Constitution de 1999. Le nom du pays sera désormais République du Venezuela, tout court ${ }^{18}$.

Ainsi, dés l'article $1^{\text {er }}$ du décret qui le sacre président, Pedro Carmona est désigné « président de la République du Venezuela » :

2. Le décret initial : 1. [...] Pedro Carmona est désigné président de la République du Venezuela, et assume par ce décret, et de manière immédiate, la fonction de chef de l'État pour la période établie par ce même décret ${ }^{19}$.

Le nouveau baptême du pays par Hugo Chávez ne fait donc pas consensus, et l'emploi du qualificatif bolivariano semble controversé.

Quant aux syntagmes revolución bolivariana et Constitución bolivariana, ils sont, somme toute, assez peu repris dans notre corpus. En effet, sur les cinquante-neuf occurrences de revolución, seulement sept sont accompagnées de l'épithète bolivariana. Et si la Constitution est mentionnée à de nombreuses reprises afin de statuer sur la légalité de la situation, on ne relève que huit occurrences du syntagme Constitución bolivariana dont trois dans un même article - auxquelles il faut ajouter deux occurrences du syntagme tronqué (voir infra). Dans les autres emplois, la Constitution est désignée - quatre fois seulement - par son véritable nom (Constitution de la République bolivarienne du Venezuela), ou par les termes « la Constitution de 1999 » et « la Constitution ».

Lorsqu'ils sont repris, on constate qu'ils sont le plus souvent mis à distance par des procédés de modalisation autonymique ${ }^{20}$. Précisons que

18. «El segundo mandato del presidente provisional fue quitar el adjetivo bolivariano establecido de la Constitución de 1999. El nombre, de ahora en adelante, será República de Venezuela, a secas », El Nacional, 13 avril 2002. Nous soulignons.

19. «El decreto inicial. 1. [...] Se designa a Pedro Carmona Estanga como presidente de la República de Venezuela, quien asume en este acto, de forma inmediata, la jefatura del Estado por el período establecido en este mismo decreto », El Universal, 13 avril 2002.

20. La modalisation autonymique est définie par Jacqueline Authier-Revuz (1995: 101) comme « modalisation consistant en une représentation réflexive opacifiante du dire en train de se faire ». Elle « se manifeste dans toute situation où l'énonciateur commente son propre dire en train de se faire; le "commentaire" témoigne d'un dédoublement au niveau de l'énonciation et peut, dans sa forme la plus réduite, se résumer à la présence de guillemets ou s'exprimer par des énoncés métadiscursifs [...] (Charaudeau et Maingueneau 2002, article «Autonymie »). 
nous employons le terme de distance à la suite de Jacqueline AuthierRevuz; autrement dit, " mettre à distance » ne doit pas être confondu « avec le fait de "prendre ses distances" vis-à-vis de mots plus ou moins rejetés comme appartenant à d'autres, qui n'est qu'une des nombreuses nuances que peut prendre la valeur fondamentale de distance inscrite dans le dire hic et nunc d'un mot, par le simple fait qu'il se réfléchisse, quelle que soit la “couleur" de ce reflet » (Authier-Revuz 1995 : 33, souligné par l'auteure). On relève ainsi deux emplois dans lesquels l'un et l'autre syntagmes sont accompagnés d'un modalisateur :

3. Sauver le Venezuela, cela symbolise le rejet face au retard que représente la prétendue révolution bolivarienne ${ }^{21}$.

4. [...] l'opinion qui prévaut est celle du spécialiste Humberto Njaim pour qui il est évident que c'est une réalité anticonstitutionnelle qui s'est imposée, même si les nouvelles autorités assoient leur légalité sur l'article 350 de la dite Constitution Bolivarienne ${ }^{22}$.

Mais les procédés de mise à distance les plus employés sont les guillemets, dont on peut observer ici un exemple pour chacun des deux syntagmes :

5. Mais, permettez-moi de citer quelques paragraphes d'un essai du poète allemand Hans Magnus Enzensberger, prémonitoires de la conduite étatique de la « révolution bolivarienne $»^{23}$.

6. Telles ont été les étapes d'un coup d'État qui de fait et de droit a annulé la validité de la Constitution « bolivarienne ${ }^{24}$.

On trouve également deux occurrences du syntagme Constitución bolivariana tronqué et guillemeté, qui devient la «bolivariana » (exemple 7), en référence, sans doute, à la manière dont Hugo Chávez désigne familièrement la Constitution.

7. L'article 233 de la « bolivarienne » prévoit qu'en cas d'empêchement absolu du président ${ }^{25}$.

21. «Rescatar a Venezuela, simboliza el rechazo ante el atraso de la pretendida revolución bolivariana », El Universal, 12 avril 2002.

22. «[...] prevalece la sentencia del estudioso Humberto Njaim para quien es evidente que lo que se ha impuesto es la realidad extraconstitucional, aún cuando las nuevas autoridades sustenta [sic] su legalidad en el artículo 350 de la llamada Constitución Bolivariana », Últimas Noticias, 13 avril 2002, «Realidad política supera normas constitucionales ».

23. «Pero, permítaseme citar unos párrafos de un ensayo del poeta alemán Hans Magnus Enserberger [sic], premonitorios de la conducta estatal de la "revolución bolivariana" », El Nacional, 12 avril 2002.

24. «Esos fueron los pasos de un golpe de Estado que de hecho y de derecho derogó la vigencia de la Constitución "bolivariana" ", El Nacional, 13 avril 2002.

25. "El artículo 233 de la "bolivariana" establece que las faltas absolutas del Presidente », El Nacional, 13 avril 2002. 
On remarque d'ailleurs que dans les emplois du syntagme complet avec guillemets, ces derniers portent sur bolivariana (exemple 6), ce qui semble indiquer que c'est la qualification de «bolivarienne » qui est mise en question; tandis que pour revolución bolivariana, c'est le syntagme entier qui est guillemeté (exemple 5). Il faut mentionner que dans les autres occurrences de revolución que l'on rencontre dans le corpus, le terme est aussi, bien souvent, mis entre guillemets ou accompagné d'un modalisateur, comme on peut le voir dans ce titre d'article (exemple 8) et dans cet extrait d'éditorial (exemple 9) :

8. Essor et chute de la «révolution ${ }^{26}$ "

9. La prétendue révolution a laissé derrière elle une autre institution ruinée et discréditée ${ }^{27}$.

Ainsi, lorsque l'expression Revolución bolivariana s'accompagne de procédés de modalisations autonymique, on peut se demander si la distance exprimée par le locuteur porte plutôt sur la qualification de « bolivarienne », sur l'utilisation par Hugo Chávez du terme de « révolution » pour désigner son processus politique, ou bien sur les deux à la fois.

Enfin, le syntagme Constitución bolivariana apparaît à deux reprises sans guillemets ni modalisation, mais dans du discours direct rapporté, donc non assumé par le journal. En définitive, sur l'ensemble du corpus, seuls trois emplois de Constitución bolivariana (exemple 10) et trois emplois de revolución bolivariana (exemple 11) sont véritablement pris en charge par le journal :

10. Chirinos ajoute que la Constitution Bolivarienne, dont il fut un des co-rédacteurs, peut être un point de départ pour la construction d'un nouveau pays ${ }^{28}$.

11. Espinoza a mené le projet de la révolution culturelle bolivarienne ${ }^{29}$.

Les syntagmes Revolución bolivariana et Constitución bolivariana, lorsqu'ils sont employés, sont donc généralement mis à distance, comme pour signaler qu'il s'agit d'expressions créées par Hugo Chávez et dont le locuteur n'assume pas la responsabilité. Ainsi, la relation établie par le président vénézuélien entre ses réalisations politiques et les valeurs associées à la figure de Simón Bolívar semble ne pas aller de soi dans le discours des journaux, en particulier dans un contexte de crise où Hugo Chávez et son gouvernement sont remis en cause.

26. «Auge y caída de la "revolución” », Últimas Noticias, 12 avril 2002.

27. "Otra institución hundida y desprestigiada dejó la pretendida revolución », El Universal, 13 avril 2002.

28. «Agrega Chirinos que la Constitución Bolivariana, de la que fue co-redactor, puede ser un punto de partida para construir un nuevo país. », El Nacional, 12 avril 2002.

29. "Espinoza llevó adelante el plan de la revolución cultural bolivariana [...]", El Nacional, 13 avril 2002. 
Enfin, le syntagme le plus employé dans notre corpus est círculos bolivarianos (cent occurrences sur cent soixante-neuf occurrences de bolivariano). Dans le discours des journaux, les cercles bolivariens sont tenus pour responsables des violences perpétrées pendant les événements, et plus précisément, des coups de feu tirés pendant la manifestation antiChávez du 11 avril 2002 et des morts qui s'ensuivirent ${ }^{30}$.

Dans l'ensemble du corpus, on relève six emplois du syntagme círculos bolivarianos accompagné d'un modalisateur («ce qu'on nomme les cercles bolivariens »), et quatre emplois du syntagme guillemeté. À deux reprises, leur nom est explicitement contesté, comme on l'observe dans ce commentaire que fait un journaliste à leur sujet :

12. Quelqu'un va devoir répondre de la filiation idéologique de toute cette violence et du fait que l'on associe le nom du héros national à des bandes armées ${ }^{31}$.

Mais dans la plupart des emplois, le segment círculos bolivarianos est pris en charge par les journaux. Il semble être passé dans le langage courant, dans la mesure où il sert à désigner un référent qui n'a pas d'autre dénomination. Par ailleurs, on constate que l'adjectif chavista se substitue parfois à l'adjectif bolivariano pour former le syntagme círculos chavistas (cercles chavistes), ou que le segment cercles bolivariens est repris par d'autres désignations, telles que « les partisans du lieutenant colonel Chávez », ou « des sympathisants du chavisme ». Ainsi, dans les emplois qui sont faits du syntagme círculos bolivarianos, l'adjectif ne semble plus indiquer de quelconque relation avec la figure de Simón Bolívar. Il s'intègre dans une expression figée, qui va servir à désigner, plus généralement, les défenseurs du régime chaviste.

\section{Vers un « oubli » du sens d'origine de bolivariano?}

Dans le deuxième type d'emploi relevé dans le corpus, l'adjectif bolivariano n'apparaît plus au sein de syntagmes figés mais dans des usages divers, que nous avons appelés emplois « libres». Ils sont au nombre d'une trentaine, et dans beaucoup de ces utilisations « libres », bolivariano s'applique aux défenseurs du régime de Chávez : « hordes bolivariennes » (« hordas bolivarianas »), " milices bolivariennes » (« milicias bolivarianas »), « groupes bolivariens armés »(« grupos bolivarianos armados »). Bolivariano semble alors devenir équivalent de chavista. On trouve d'ailleurs certains emplois où le substantif qualifié de « bolivarien » est précédé d'un adjectif possessif, ce qui fait apparaître de manière encore plus explicite la relation avec Hugo Chávez :

30. On notera que cette accusation est contestable, ces assassinats ayant été attribués par la suite à des francs-tireurs dont l'identité est encore incertaine.

31. «Alguien va a tener que responder por la vertebración ideológica de toda esta violencia y de que asociemos el nombre del héroe nacional con bandas armadas », El Universal, 13 avril 2002. 
13. [...] de la main de ses francs-tireurs bolivariens ${ }^{32}$.

14. Le président, [...] dans ses propres syndicats bolivariens, contrôlés par l'État ${ }^{33}$.

On relève également quelques emplois de bolivariano substantivé, dans lesquels ce dernier est toujours opposé au terme escuálido (maigrichon, décharné), surnom donné par Hugo Chávez à ses opposants :

15. Lorsque j'ai vu que la GN [la Garde Nationale] était en train de protéger l'un des groupes (les bolivariens) contre les manifestants pacifiques (les escuálidos $[\ldots]^{34}$.

16. Comme si tout un chacun - catégorisé comme "escuálido» ou « bolivarien »-avait trouvé une case, une certitude, et même un orgueil $1^{35}$.

Ces emplois semblent témoigner d'une évolution de sens de l'adjectif bolivariano. Il paraît indiquer désormais une appartenance politique : dans le Venezuela d'Hugo Chávez, on est soit bolivariano, soit escuálido.

On trouve enfin des emplois assez saisissants, tels que l'expression « crise bolivarienne » pour désigner la crise politique qui touche le pays, autrement dit la crise du gouvernement de Chávez; ou la description d'un partisan d'Hugo Chávez, " camouflé derrière des lunettes noires et une casquette bolivarienne ${ }^{36}$ ", en référence, sans doute, au fameux béret rouge du président vénézuélien. Dans ces utilisations « libres », l'adjectif bolivariano perd son signifié d'origine, il n'indique plus désormais une relation - quelle qu'elle soit - avec Simón Bolívar, mais avec Hugo Chávez et sa politique « bolivarienne ».

Pour conclure, on a donc pu constater que l'adjectif bolivariano est un adjectif ambigu de par sa morphologie même, et que ses emplois en contexte ne permettent pas forcément de le désambiguïser. C'est sans doute cette non-univocité qui permet à Hugo Chávez d'en généraliser l'usage au point de l'employer dans les contextes les plus singuliers. Dans l'usage qu'en fait le président vénézuélien, l'adjectif bolivariano permet de mobiliser les valeurs positives associées dans la mémoire collective à la figure mythique de Simón Bolívar. En circulant à travers d'autres discours, il conserve la trace de ces emplois, et renvoie alors au discours d'Hugo Chávez. La presse, en reprenant cet adjectif, adopte ainsi une double attitude. Soit elle met à distance les syntagmes créés par Hugo Chávez; dans ce cas, la qualification

32. « [...] a manos de sus francotiradores bolivarianos », El Nacional, 12 avril 2002.

33. «El presidente, $[\ldots]$ en sus propios sindicatos bolivarianos, controlados por el Estado», « Hugo Chávez : un autócrata en substancia, no sólo en estilo », El Universal, 12 avril 2002. 34. "Cuando vi que la GN estaba protegiendo a un grupo (los bolivarianos) en contra de los manifestantes pacíficos (los escuálidos)», Últimas Noticias, 15 avril 2002.

35. «Como si cada quien -dividido en "escuálido" o en "bolivariano"- hubiese encontrado una casilla, una certeza, y hasta un orgullo », El Nacional, 15 avril 2002.

36. «Camuflado detrás de lentes oscuros y gorra bolivariana », El Nacional, 12 avril 2002. 
de bolivariano/a semble poser problème et l'adjectif devient alors lieu de débats. Soit les journaux intègrent le terme bolivariano dans leur discours, sans le remettre en cause, mais pour lui donner un nouveau signifié : celuici indique alors une relation, non plus avec le personnage de Bolívar, mais avec la figure politique d'Hugo Chávez. En ce sens, ne peut-on pas parler de phénomène de dé-mémoire discursive, au sens où l'entend Marie-Anne Paveau (2006 : 110-116)? En effet, dans le premier cas de figure, les rédacteurs des journaux remettent en question le fait que Chávez qualifie ses réalisations politiques de « bolivariennes ». Ils semblent donc juger qu'en les qualifiant ainsi, Hugo Chávez « oublie » le sens d'origine - ou le sens « véritable » - du terme « bolivarien ». Dans le second cas de figure, ce n'est plus un jugement d'oubli, mais un oubli pur et simple, de la part de ces rédacteurs, du fait que bolivariano est lié à l'origine au personnage de Bolívar et non à la « révolution bolivarienne » d'Hugo Chávez. S'il y a phénomène de dé-mémoire, c'est donc au profit d'une nouvelle mémoire plus récente, celle des discours circulant dans l'ère Chávez.

Églantine Samouth Université Paris Est Créteil-CEDITEC

\section{Références}

Arraíz, A., 1940, Culto bolivariano, Caracas, Editorial Cóndor.

Barrera Linares, L., 2003, « Discurso y comportamiento venezolanos : "sociedad civil" contra "círculos bolivarianos" ", Discurso y Sociedad, vol. 4, n 3, Discurso y democracia en Venezuela, p. 57-76.

Bosque, I. et Demonte V. (dir.), 1999, Gramática descriptiva de la lengua española, t. III : Entre la oración y el discurso. Morfología, Madrid, Espasa, Real Academia Española, coll. « Nebrija y Bello ».

Carrera Damas, G., 1989, El culto a Bolivar, Caracas, Grijalbo.

Castillero, E. J., [1948] 1995, Historia de Panamá, Panamá, Lic. Eduardo, $11^{\text {e éd. }}$ Charaudeau, P. et Maingueneau, D. (dir.), 2002, Dictionnaire d'analyse du discours, Paris, Seuil.

Chumaceiro Arreaza, I., 2003, «El discurso de Hugo Chávez : Bolívar como estrategia para dividir a los venezolanos », Boletín de Lingüística, $\mathrm{n}^{\circ} 20$, http:// redalyc.uaemex.mx/redalyc/src/inicio/ArtPdfRed.jsp ?iCve=34702003.

Jonasson, K., 1994, Le Nom propre. Constructions et interprétations, Louvain-la-Neuve, Duculot. 
Krieg, A., 2002, «L'adjectif “ethnique” entre langue et discours. Ambiguïté relationnelle et sous-détermination énonciative des adjectifs dénominaux », RSP Revue de sémantique et pragmatique, $\mathrm{n}^{\circ}$ 11, p. 103-121.

Lecolle, M., Paveau M.-A. et Reboul-Touré S., 2009, « Avant-propos. Le sens des noms propres en discours », Les Carnets du Cediscor, no 11 , Le Nom propre en discours.

Lignon, St., 2002, « L'adjectif en -ien comme révélateur de phénomènes de concurrence ", Bulletin de linguistique appliquée et générale, $\mathrm{n}^{\circ} 27$, http://w3.erss.univ-tlse2.fr/textes/publications/CE/Lignon.pdf.

Maingueneau, D., 1991, L'Analyse du discours. Introduction aux lectures de l'archive, Paris, Hachette.

Moirand, S., 2007, «Discours, mémoires etcontextes: à propos du fonctionnement de l'allusion dans la presse », Corela, Numéros spéciaux, Cognition, discours, contextes, http://edel.univ-poitiers.fr/corela/document.php?id=1636.

Paveau, M.-A., 2006, Les Prédiscours. Sens, mémoire, cognition, Paris, Presses Sorbonne Nouvelle.

Quintero, I., 2000, « Del Bolívar para todos al Bolívar para Chávez », Espaces latinos, ${ }^{\circ} 171$, p. 15-16.

Riegel, M. et al., 1994, Grammaire méthodique du français, Paris, PUF.

Siblot, P., 1987, « De la signifiance du nom propre », Cahiers de praxématique, $\mathrm{n}^{\circ}$ 8, Praxiling, Publications de l'université Paul-Valéry, Montpellier 3, p. 97-114.

Siblot, P. et Leroy, S., 2000, " L'antonomase entre nom propre et catégorisation nominale ", Mots. Les langages $d u$ politique, $\mathrm{n}^{\circ}$ 63, p. 89-104, http:/www.persee.fr/web/revues/home/prescript/article/ mots_0243-6450_2000_num_63_1_2207.

Wilmet, M., 1995, « Pour en finir avec le nom propre », L'information grammaticale, $\mathrm{n}^{\circ}$ 92, p. 113-124.

\section{Dictionnaires}

Diccionario de la lengua española, 1992, Madrid, Real Academia española. Dictionnaire en ligne : http://www.rae.es.

Diccionario de uso del español, 1998, Moliner María, Madrid, Gredos.

Diccionario etimológico español e hispánico, 1955, García de Diego Vicente, Madrid, SAETA.

Enciclopedia del idioma : diccionario histórico y moderno de la lengua española (siglos XII al XX) etimológico, tecnológico, regional e hispanoamericano, 1958, Alonso Pedraz Martín, Madrid, Aguilar.

Nuevo diccionario de americanismos e indigenismos, 1998, Marcos A. Morínigo, (version actualisée par Marcos Alberto Morínigo VázquezPrego), Buenos Aires, Claridad. 
